\title{
Anomalia de Ebstein: um estudo de caso
}

\author{
Ebstein's anomaly: a case study \\ La anomalía de Ebstein, un estudio de caso
}

Rhaiane Evelyn Simmer Schunk ${ }^{1}$, Rhainer Victor Simmer Schunk ${ }^{1}$, Amanda Rodrigues Lessa ${ }^{1 *}$, Guilherme Rodrigues Lessa', Ana Carolina Rocha Auer ${ }^{1}$, Cesar Quintaes Freitas Lima Filho², Melchior Luiz Lima², Héber Souza Melo Silva².

\section{RESUMO}

Objetivo: Estudar, a partir da literatura, um caso de Anomalia de Ebstein, expondo as características clínicas apresentadas no momento e a evolução do paciente, no estado do Espírito Santo. Detalhamentos do Caso: Masculino, 62 anos, buscou o serviço especializado queixando-se de fadiga e dispneia. Histórico patológico pregresso de hipertensão, obesidade, diabetes, insuficiencia cardíaca, dextrocardia, cardiomegalia, anomalia de Ebstein, fibrilação atrial paroxística e infarto agudo do miocárdio. Histórico familiar de cardiopatia e hábitos de etilismo e fumo. Na admissão apresentava-se com dispneia progressiva classificação funcional NYHA IV. Ao ecocardiograma transtorácico, aumento importante de câmaras direitas, disfunção diastólica grau I e anomalia de Ebstein. Foi operado com troca valvar tricúspide (prótese biológica no33) e cirurgia de revascularização do miocárdio em descendente anterior, com tempo de circulação extra-corpórea em 142 minutos, sem intercorrências, evoluindo bem e recebendo alta. Atualmente paciente encontra-se em casa, em acompanhamento. Considerações Finais: A anomalia de Ebstein é uma cardiopatia congênita, geralmente conduzida e corrigida na infância, entretanto, há alguns casos individualizados em adultos. Seu manejo deve ser diferenciado conhecendo suas distinções anatômicas, variáveis hemodinâmicas e morbidades associadas, categóricos para um bom manejo do seu portador, revigorando sua qualidade de vida.

Palavras-chave: Anomalia de Ebstein, Cardiologia, Cirurgia cardíaca.

\begin{abstract}
Objective: To study, based on the literature, a case of Ebstein's Anomaly, exposing the clinical characteristics presented at the time and the patient's evolution, in the state of Espírito Santo. Case Details: Male, 62 years old, sought the specialized service complaining of fatigue and dyspnea. Past pathological history of hypertension, obesity, diabetes, heart failure, dextrocardia, cardiomegaly, Ebstein's anomaly, paroxysmal atrial fibrillation, and acute myocardial infarction. Family history of heart disease and drinking and smoking habits. On admission, he presented with progressive dyspnea NYHA IV functional classification. Transthoracic echocardiogram showed significant enlargement of the right chambers, grade I diastolic dysfunction and Ebstein's anomaly. He was operated on with tricuspid valve replacement (biological prosthesis No. 33 ) and coronary artery bypass graft surgery in the anterior descending, with cardiopulmonary bypass time of 142 minutes, without complications, progressing well and being discharged. Currently, the patient is at home, under follow-up. Final Considerations: Ebstein's anomaly is a congenital heart disease, usually managed and corrected in childhood, however, there are some individualized cases in adults. Its management must be differentiated, knowing its anatomical distinctions, hemodynamic variables, and associated morbidities, categorical for a good management of its carrier, reinvigorating their quality of life.
\end{abstract}

Key words: Ebstein's anomaly, Cardiology, Cardiac surgery.

1 Universidade Vila Velha (UVV), Vila Velha - ES. *E-mail: amanda.r-l@hotmail.com

2 Hospital Meridional, Cariacica - ES. 


\section{RESUMEN}

Objetivo: Estudiar, con base en la literatura, un caso de Anomalía de Ebstein, exponiendo las características clínicas presentadas en el momento y la evolución del paciente, en el estado de Espírito Santo. Detalle del caso: Varón, 62 años, acude al servicio especializado por fatiga y disnea. Antecedentes patológicos de hipertensión, obesidad, diabetes, insuficiencia cardíaca, dextrocardia, cardiomegalia, anomalía de Ebstein, fibrilación auricular paroxística e infarto agudo de miocardio. Antecedentes familiares de enfermedades cardíacas, hábitos de beber y fumar. Al ingreso presentó disnea progresiva de clasificación funcional NYHA IV. El ecocardiograma transtorácico mostró agrandamiento significativo de cavidades derechas, disfunción diastólica grado I y anomalía de Ebstein. Fue intervenido con recambio valvular tricúspide (prótesis biológica no 33) y cirugía de revascularización coronaria en descendente anterior, con tiempo de circulación extracorpórea de 142 minutos, sin complicaciones, con buena evolución y alta. Actualmente, el paciente se encuentra en casa, en seguimiento. Consideraciones finales: La anomalía de Ebstein es una enfermedad cardíaca congénita, generalmente manejada y corregida en la niñez, sin embargo, existen algunos casos individualizados en adultos. Su manejo debe ser diferenciado, conociendo sus distinciones anatómicas, variables hemodinámicas y morbilidades asociadas, categóricas para un buen manejo de su portador, dinamizando su calidad de vida.

Palabras clave: Anomalía de Ebstein, Cardiología, Cirugía cardíaca.

\section{INTRODUÇÃO}

Trata-se de uma malformação congênita rara, caracterizada por anormalidades da valva tricúspide e do Ventrículo Direito (VD), clinicamente variável, desde uma condição crítica em neonatos até adultos assintomáticos. O diagnóstico é confirmado, ao Ecocardiograma Transtorácico (ECOTT), com o deslocamento apical do folheto septal da válvula tricúspide em $\geq 8 \mathrm{~mm} / \mathrm{m} 2 \mathrm{em}$ comparação com o folheto anterior da válvula mitral. A farmacoterapia inclui diuréticos, controle de ritmo e terapia para disfunção sistólica ventricular esquerda. $O$ tratamento cirúrgico é indicado em casos de insuficiência tricúspide acentuada (classe NYHA III ou IV) ou deterioração da capacidade funcional (YUAN SM, 2017; CONNOLLY MH e QURESHI MY, 2020; HOLST KA, et al., 2019).

A ecocardiografia transtorácica é o exame usado para o diagnóstico, avaliação anatômica inicial e acompanhamento da anomalia. Também é recomendado imagens de Ressonância Magnética Cardiovascular visto que ela fornece informações complementares às obtidas na ecocardiografia, incluindo a quantificação do tamanho e função do ventrículo direito e visualização da anatomia da valva tricúspide (QUERESHI MY, et al., 2019; YANG D, et al., 2018; NEIJENHUIS RML, et al., 2021).

A abordagem para tratamento é determinada pela apresentação clínica e idade do paciente, sendo que os componentes do manejo incluem o monitoramento, gerenciamento médico e de arritmias, intervenção cirúrgica ou por cateter. Muitos pacientes são assintomáticos e necessitam apenas de monitoramento, enquanto outros apresentam sintomas e requerem terapia médica de suporte e intervenção cirúrgica. Todos os pacientes devem ser submetidos a monitoramento regular para determinar a necessidade de terapia médica, cirurgia ou intervenção por cateter ou reintervenção cirúrgica (STOUT KK, et al., 2019).

Posto isso, objetivamos estudar, a partir da literatura, um caso de Anomalia de Ebstein, expondo as características clínicas apresentadas no momento e a evolução do paciente, no estado do Espírito Santo.

\section{DETALHAMENTO DO CASO}

O presente artigo foi conduzido como um estudo de caso observacional e descritivo. Para fins legais, foi aplicado o Termo de Consentimento Livre e Esclarecido (TCLE), elaborado de forma a elucidar o objetivo do estudo, a importância da participação do paciente, e a manutenção do anonimato a partir do aceite de colaboração por ele. Foram esclarecidas todas as questões éticas, os riscos e as dúvidas. O documento foi formulado em duas cópias, lido e assinado pelo participante e pelo pesquisador. 
J.L.A, 63 anos, casado há 30 anos, professor aposentado, residente de Vitória, Espírito Santo. Internou em serviço especializado (Unidade Coronariana) no dia 16/08/2020 apresentando um quadro de dispneia progressiva Classificação Funcional NYHA IV. Histórico patológico pregresso de Hipertensão Arterial Sistêmica (HAS), Diabetes Mellitus (DM), Insuficiência Cardíaca (IC), obesidade mórbida, dextrocardia, cardiomegalia, Anomalia de Ebstein, Fibrilação Atrial Paroxística e insuficiência venosa, infarto do miocárido descoberto através de cateterismo datado entre 2000 e 2002, cirurgia de prótese de fêmur por queda de própria altura e retirada de veia safena direita em 2019. Nega alergias.

Possui histórico familiar de cardiopatia e hábitos de etilismo e fumo. Relata ter parado de fumar há 15 anos, chegando a fumar 20 maços/dia. Em uso de Diovan HCT 320/25mg, Concardio 5mg, Ácido Acetilsalicílico $100 \mathrm{mg} \mathrm{1x/dia,} \mathrm{Apixaban} \mathrm{4mg} \mathrm{2x/dia,} \mathrm{Crestor} 20 \mathrm{mg} \mathrm{1x/dia,} \mathrm{Furosemida} \mathrm{40mg} \mathrm{2x/dia,}$ Espironolactona 25mg 1x/dia, Glifage XR500 2x/dia, Dapglifozina 10mg 1x/dia, Alopurinol 300mg, Trazodona $50 \mathrm{mg} 1 \mathrm{x} / \mathrm{dia}$, Zolpidem $10 \mathrm{mg} 1 \mathrm{x} / \mathrm{dia}$ e uso tópico de óxido zinco + nistatina $3 \mathrm{x} / \mathrm{dia}$, cetoconazol $2 \% 30 \mathrm{~g} 3 \mathrm{x} / \mathrm{dia}$ e dexametasona creme $0,1 \% 3 x /$ dia

Ao exame físico, frequência cardíaca (FC): 93 bpm, Pressão Arterial (PA): 101/52 mmHg, Murmúrio Vesicular Fisiológico (MVF) reduzido bilateralmente em bases, saturando $96 \%$, abdome globoso de difícil avaliação devido a adiposidade aumentada, MMII apresentando dermatite ocre, linfedema crônico e celulite em MMI (em tratamento com Doxiciclina desde 13/08/2020), panturrilhas livres.

Os exames de imagem evidenciaram radiografia de tórax apresentando Dextrocardia (Figura 1) com cardiomegalia e congestão pulmonar, USG de abdome com hepatopatia congestiva e aumento de calibre de veias supra-hepáticas e cava inferior.

Figura 1 - Radiografia de Tórax evidenciando dextrocardia e cardiomegalia.

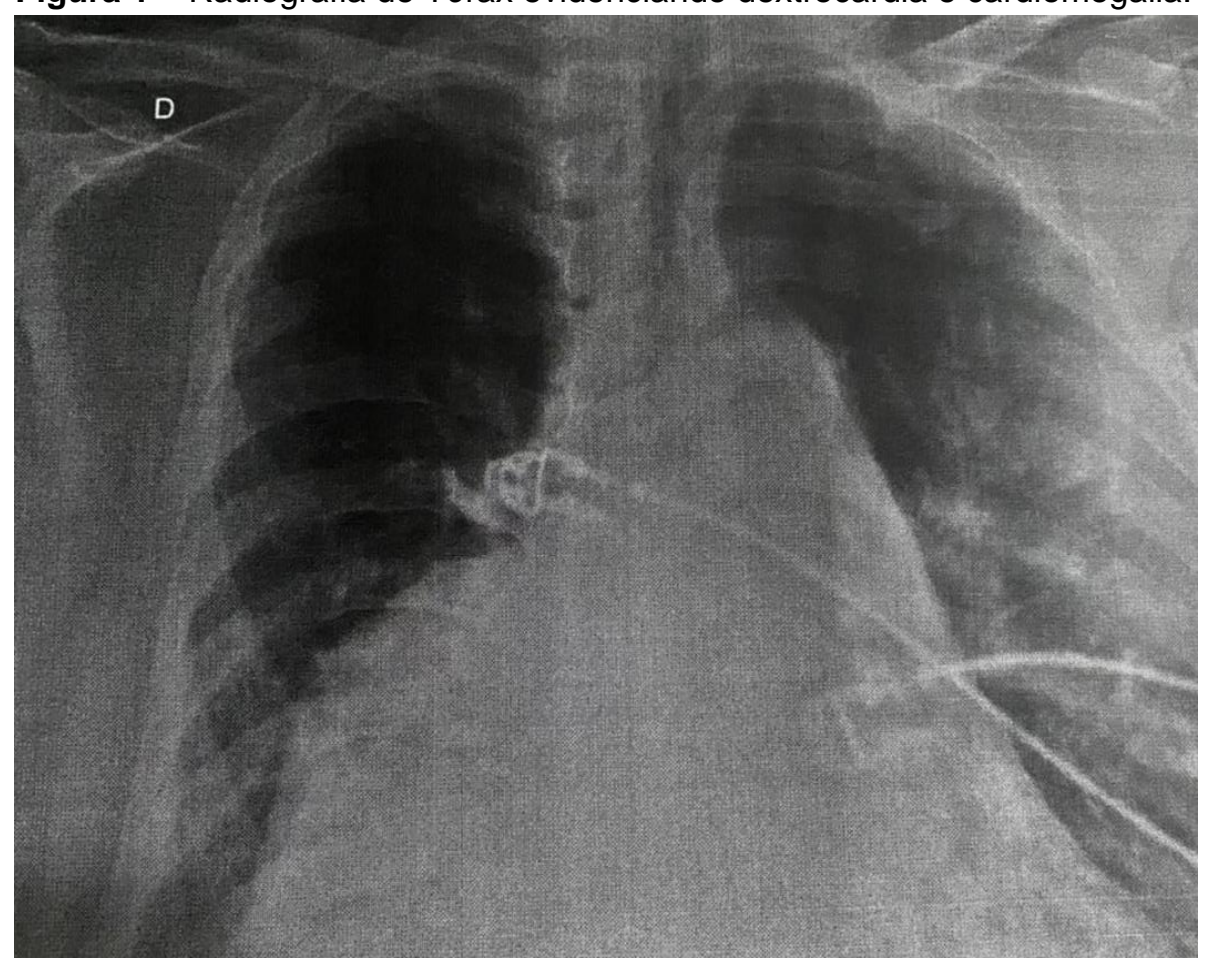

Fonte: Schunk RES, et al., 2021.

Ao Ecocardiograma Transtorácico (ETT) em 05/08/2020 (trazido pelo paciente): átrio esquerdo (AE) 40mm, ventrículo esquerdo (VE) 9/9/48/37, fração de ejeção (FE) $63 \%$, ventrículo direito (VD) dilatado $(50 \mathrm{~mm})$, Dextrocardia, VE com função preservada, anomalia de Ebstein com insuficiência de tricúspide importante (IT), VD e átrio direito (AD) dilatados e hipocontrátil, pressão sistólica de artéria pulmonar (PSAP) $50 \mathrm{mmHg}$. 
$\mathrm{Na}$ internação foi realizado Doppler Venoso de MMII que identificou junção safeno-femoral esquerda incompetente, sem trombose evidente; Doppler Arterial de MMII evidenciando ateromatose do segmento fêmuro-poplíteo tibial sem estenose significativa bilateralmente e Doppler de Carótidas demonstrando estenose bilateral de 20\%. Em 25/08/2020 ETT: artéria aorta (AO) $24 \mathrm{~mm}$ diâmetro diastólico, AE 37mm, VE 43/27/9/9, FE 62\% (aumento importante de câmaras direitas), disfunção diastólica grau I, IT importante, PSAP $45 \mathrm{mmHg}$.

Em 26/08/2020 foi realizado cateterismo evidenciando oclusão de artéria descendente anterior com colateral bem desenvolvida de múltiplas origens. No dia 27/08/2020 CATE (comunicado equipe para troca valvar e revascularização). Iniciado dobutamina $10 \mathrm{ml} / \mathrm{h}(5 \mathrm{mcg} / \mathrm{kg} / \mathrm{min}) \mathrm{com}$ posterior desmame no mesmo dia. Aguardando melhora da função renal para programação de plastia mitral e revascularização (entre 27/08/2020 até 07/09/2020).

Pós-operatório imediato de troca valvar tricúspide (prótese biológica №33) + cirurgia de revascularização miocárdica da artéria mamária-DA em 08/09/2020. Tempo de circulação extracorpórea de 142 minutos, sem necessidade de concentrado de hemácias e plaquetas. Paciente chegou da cirurgia instável hemodinamicamente em uso de dobutamina $60 \mathrm{ml} / \mathrm{h}(10 \mathrm{mcg} / \mathrm{kg} / \mathrm{min})$, noradrenalina $30 \mathrm{ml} / \mathrm{h}(0.3 \mathrm{mcg} / \mathrm{kg} / \mathrm{min})$, nitroprussiato sódico $5 \mathrm{ml} / \mathrm{h}$, em IOT/VM/PCV, permanecendo em uso de drogas vasoativas até dia 15/09/2020.

Ecocardiograma Transesofágico (ETE) após procedimento: VE com tamanho e função preservado. VD apresenta aumento moderado e hipocinesia de grau moderado. Prótese biológica em posição tricúspide com abertura e movimentação normal de seus folhetos. Fluxo laminar através da valva. Ausência de regurgitação central ou periprotética. Insuficiência mitral de grau discreto. Em ETT 10/09/2020: VE normal, VD com disfunção leve (milrinona + dobutamina). Prótese tricúspide normofuncionante (graduação máxima 4 e médio 2). Sem derrame pericárdico (janela ainda limitada)

Tomografia computadorizada (TC) em 16/09/2020 mostrando pulmões pouco insuflados com densidade difusamente heterogênea, opacidades em vidro fosco associado a espessamento regular dos septos interlobulares de forma difusa, com predomínio medular, sugerindo congestão pulmonar. Moderado/volumoso derrame pericárdico. Dextrocardia com aumento da área cardíaca. ETE com VE normal, VD com importante disfunção, prótese normofuncionante, imagem sugerindo derrame pericárdico com muita fibrina e trabeculações.

Sendo realizado drenagem de pericárdio (material organizado/líquido/coágulo e fibrina) no dia seguinte, com cultura de secreção mediastinal negativa. Eletrocardiograma (ECG) com taquicardia atrial/flutter com alto grau de bloqueio. Exames laboratoriais não demonstraram alterações, não houve sinais infecciosos e função renal estável no momento.

Em 22/09/2020 foi implantado marcapasso bicameral definitivo (procedimento tecnicamente difícil e demorado devido Anomalia de Ebstein e dextrocardia do paciente). Paciente evolui pós procedimento de forma hemodinamicamente estável em baixas doses de nitroprussiato de sódio, ferida operatória sem sinais flogísticos, lúcido e orientado em tempo e espaço, interagindo com o examinador, sem déficits neurológicos ou focais, pupilas isocóricas e fotorreagentes.

Apresentava ritmo cardíaco regular em dois tempos, bulhas hipofonéticas sem sopro audível, PA 130/68mmHg, PA média $89 \mathrm{mmHg}$, FC 78bpm no comando de marcapasso, presença de dreno Blake de mediastino com conteúdo hemático; MVF sem ruídos adventícios, sem esforço respiratório, saturando 94\% com cateter nasal de oxigênio a 2L/min, intervalando com ventilação não invasiva sem intercorrências; diurese presente quantificada por sonda vesical de demora e com bom débito urinário; abdome globoso, flácido, sem visceromegalias ou massas palpáveis, indolor a palpação e aceitando dieta oral, evacuação presente e glicemia controlada; exames laboratoriais sem alterações, sem febre nas últimas 24 horas e em uso de vancomicina e cefepime (dia 6/7)

Tomografia de tórax (TC) em 28/09/2020: em análise comparativa a exame anterior datado de 16/09/2020, destaca-se: introdução de dreno pericárdico a esquerda com resolução praticamente total da coleção nessa 
topografia. Aumento das atelectasias/consolidações posteriores nos pulmões. A conduta a partir do dia 28/09/2020, uma vez que o paciente evoluía favoravelmente, foi seguir com reabilitação, sentar-se fora do leito, deambular com auxílio, evoluindo com alta definitiva em 09/10/2020. Já em seu domicílio em 22/10/2020 foi constatado que constatamos ele evoluía bem, sem intercorrências ou queixa e estava em acompanhamento ambulatorial.

\section{DISCUSSÃO}

Uma vez que alguns dados científicos acerca da Anomalia de Ebstein ainda são um pouco escassos, expandimos o recorte temporal para apresentarmos os achados epidemiológicos do nosso estado, esperando contribuir com outros estudos desta natureza. Trata-se de um defeito na Válvula Tricúspide com risco estimado na população em geral de 1 em 20.000 nascidos vivos, sem predileção por nenhum dos sexos (LUPO PJ, et al., 2011; CORREA-VILLASEÑOR A, et al., 1994; PRADAT P, et al., 2003).

A predisposição genética para anomalia de Ebstein é considerada heterogênea. Nesta anomalia os pontos de ancoragem dos folhetos da tricúspide estão deslocados dentro do Ventrículo Direito. Como resultado, o folheto septal fica encurtado enquanto o folheto anterior sofre ampliação, o átrio direito fica bem maior que o normal e também é comum encontrar Forame Oval Patente (FOP) ou Defeito do Septo Atrial (DSA). A válvula anormal pode provocar regurgitação para o átrio direito e a regurgitação somada a um pequeno VD efetivo pode provocar falência cardíaca. Também é possível que ocorra shunt sanguíneo do Átrio Direito $(A D)$ para o Átrio Esquerdo (AE) através do FOP/DSA. O ventrículo direito atrializado geralmente tem paredes finas e demonstra baixa contratilidade (BENSON DW, et al., 1999; KELLE AM, et al., 2016; POSTMA AV, et al., 2011; VAN ENGELEN K, et al., 2013; ATTENHOFER JOST CH, et al., 2005).

A apresentação clínica varia amplamente. Em geral, os sintomas estão relacionados ao grau de anormalidade anatômica e a magnitude do shunt interatrial da direita para a esquerda. Pode ser fatal no útero ou logo após o nascimento se houver cardiomegalia grave, hipoplasia pulmonar devido a cardiomegalia maciça ou insuficiência cardíaca (QUERESHI MY, et al., 2018).

Indivíduos com deslocamento acentuado da tricúspide podem, desde a infância até a fase adulta, desenvolver regurgitação grave, insuficiência cardíaca direita, pressão atrial direita elevada e cianose acentuada. Estes acometimentos são devidos ao shunt interatrial direito-esquerdo. Por outro lado, pacientes com deslocamento apical menos acentuado ou que apresentem alterações anatômicas brandas podem permanecer assintomáticos durante a idade adulta (QUERESHI MY, et al., 2019).

Adicionalmente, a idade precoce de manifestação foi frequentemente associada a outras lesões cardíacas, particularmente defeito do septo atrial e estenose pulmonar, que predispõem à cianose por conta do shunt da direita para a esquerda e a apresentação clínica também variou de acordo com a idade no momento diagnóstico, endossando a intervenção e o manejo precoces como forma de contenção dos agravos esperados (QUERESHI MY, et al., 2019).

Deve-se suspeitar de anomalia de Ebstein em bebês, crianças e adultos cianóticos, também em indivíduos que apresentem regurgitação tricúspide com ou sem insuficiência cardíaca e naqueles que apresentam embolia sistêmica paradoxal ou arritmias. O diagnóstico pode ser suspeitado clinicamente, mas é confirmado pela identificação de deslocamento apical da inserção do folheto da válvula tricúspide septal em $\geq 8 \mathrm{~mm} / \mathrm{m} 2$ indexado pela área de superfície corporal em comparação com a inserção do folheto da válvula mitral anterior. Isso é mais comumente demonstrado na visão ecocardiográfica transtorácica apical de quatro câmaras (QERESHI MY, et al., 2018).

A ecocardiografia transtorácica é geralmente o exame chave para o diagnóstico, avaliação anatômica inicial e acompanhamento da anomalia de Ebstein. Também é comumente recomendado imagens de Ressonância Magnética Cardiovascular porque ela fornece informações complementares às obtidas na ecocardiografia, incluindo a quantificação do tamanho e função do ventrículo direito, além da visualização da anatomia da valva tricúspide (QUERESHI MY, et al., 2019; YANG D, et al., 2018; NEIJENHUIS RML, et al., 2021). 
A abordagem para tratamento é determinada pela idade do paciente e apresentação clínica. Os componentes do manejo são monitoramento, gerenciamento médico (incluindo alívio temporário dos sintomas antes da cirurgia), gerenciamento de arritmias e intervenção cirúrgica ou por cateter. Muitos pacientes não apresentam sintomas e requerem apenas monitoramento, enquanto outros são sintomáticos e requerem terapia médica de suporte e intervenção cirúrgica. Todos os pacientes devem ser submetidos a monitoramento periódico para determinar a necessidade de terapia médica, cirurgia ou intervenção por cateter ou reintervenção cirúrgica (STOUT KK, et al., 2019).

O intervalo de tempo para monitoramento varia com a gravidade da doença e o estágio fisiológico do paciente. Para pacientes assintomáticos, a recomendação é o monitoramento a cada 12 meses. Para pacientes com regurgitação tricúspide leve a moderada, recomenda-se avaliação a cada um a dois anos. Avaliação adicional é indicada se o paciente desenvolver novos sinais ou sintomas cardiovasculares.

O monitoramento deve incluir avaliação clínica, avaliação de arritmias, eletrocardiograma, ecocardiografia transtorácica abrangente e ressonância magnética cardíaca. Em adultos, a RMC é recomendada a cada um a dois anos em pacientes sintomáticos e a cada três a cinco anos em pacientes assintomáticos. Quanto ao gerenciamento médico, segundo a literatura, são categorizados os tipos de abordagem especificamente para neonato sintomático, criança sintomática, adulto sintomático, profilaxia contra tromboembolismo e endocardite, arritmias e abordagem gestacional (STOUT KK, et al., 2019). A correção cirúrgica deve ser evitada no período neonatal, pois o risco de mortalidade é alto (10 a 25 por cento) (KNOTT-CRAIG CJ, et al., 2007, KNOTT-CRAIG CJ, et al., 2008; STARNES VA, et al., 1991).

Para a maioria dos neonatos sintomáticos o manejo médico de suporte com terapia intensiva neonatal avançada é preferível com o objetivo de atrasar a cirurgia o máximo possível. Contudo, a cirurgia pode ser necessária para aqueles que não conseguem ser retirados do ventilador ou apresentam anormalidades metabólicas persistentes. Para intervenção cirúrgica ou cateterização em adultos e crianças existem numerosos critérios de acordo com as diretrizes de doenças cardíacas congênitas adultas da American College of Cardiology/American Heart Association de 2018, o que inviabiliza uma menção sucinta (STOUT KK, et al., 2019).

A anomalia de Ebstein é uma malformação cardíaca congênita complexa, com grande diferença de apresentação anatômica e clínica. Sua gestão precisa ser particularizada. Suspeita-se de anomalia de Ebstein em pacientes cianóticos, com regurgitação tricúspide, insuficiência cardíaca e nas arritmias. Conhecer suas características anatômicas, variáveis hemodinâmicas e malformações associadas são decisivos para um bom manejo, otimizando, assim, a longevidade do paciente.

\section{REFERÊNCIAS}

1. ATTENHOFER JOST CH, et al. Left heart lesions in patients with Ebstein anomaly. Mayo Clin Proc, 2005; 80:361.

2. BENSON DW, et al. Mutations in the cardiac transcription factor NKX2.5 affect diverse cardiac developmental pathways. J Clin Invest, 1999; 104:1567.

3. CONNOLLY HM, et al. Clinical manifestations and diagnosis of Ebstein anomaly. UpToDate, 2020.

4. CONNOLLY HM, et al. Management and prognosis of Ebstein anomaly. UpToDate, 2019.

5. CORREA-VILLASEÑOR A, et al. Ebstein's malformation of the tricuspid valve: genetic and environmental factors. The Baltimore-Washington Infant Study Group. Teratology, 1994; 50: 137.

6. HOLST KA, et al. Ebstein's Anomaly. Methodist Debakey Cardiovasc J, 2019; 15(2): 138-144.

7. KELLE AM, et al. Ebstein anomaly, left ventricular non-compaction, and early onset heart failure associated with a de novo a-tropomyosin gene mutation. Am J Med Genet A, 2016; 170: 2186.

8. KNOTT-CRAIG CJ, et al. Repair of neonates and young infants with Ebstein's anomaly and related disorders. Ann Thorac Surg, 2007; 84: 587.

9. KNOTT-CRAIG CJ, et al. Surgical strategy to prevent cardiac injury during reoperation in infants. J Cardiothorac Surg, 2008; 3: 10.

10. LUPO PJ, et al. Epidemiology of Ebstein anomaly: prevalence and patterns in Texas, 1999-2005. Am J Med Genet A, 2011; 155A: 1007.

11. NEIJENHUIS RML, et al. Cone reconstruction for Ebstein anomaly: Late biventricular function and possible remodeling. J Thorac Cardiovasc Surg, 2021; 161: 1097. 
12. POSTMA AV, et al. Mutations in the sarcomere gene MYH7 in Ebstein anomaly. Circ Cardiovasc Genet. $2011 ; 4: 43$.

13. PRADAT $P$, et al. The epidemiology of cardiovascular defects, part I: a study based on data from three large registries of congenital malformations. Pediatr Cardiol, 2003; 24: 195.

14. QURESHI MY, et al. Cardiac imaging in Ebstein anomaly. Trends Cardiovasc Med 2018; $28: 403$.

15. QURESHI MY, et al. Tricuspid Valve Imaging and Intervention in Pediatric and Adult Patients With Congenital Heart Disease. JACC Cardiovasc Imaging, 2019; 12: 637.

16. STARNES VA, et al. Ebstein's anomaly appearing in the neonate. A new surgical approach. J Thorac Cardiovasc Surg, 1991; 101: 1082.

17. STOUT KK, et al. 2018 AHA/ACC Guideline for the Management of Adults With Congenital Heart Disease: A Report of the American College of Cardiology/American Heart Association Task Force on Clinical Practice Guidelines. Circulation, 2019; 139: e698.

18. VAN ENGELEN K, et al. Ebstein's anomaly may be caused by mutations in the sarcomere protein gene MYH7. Neth Heart J, 2013; 21: 113.

19. YANG D, LI X, et al. Cardiovascular magnetic resonance evidence of myocardial fibrosis and its clinical significance in adolescent and adult patients with Ebstein's anomaly. J Cardiovasc Magn Reson, 2018; 20: 69.

20. YUAN SM. Ebstein's Anomaly: Genetics, Clinical Manifestations, and Management. Pediatr Neonatol, 2017; 58(3): 211-215. 